\title{
Mechanically expanding transcatheter aortic valves: pros and cons of a unique device technology
}

\author{
Kenan Yalta, Muhammet Gurdogan, Cafer Zorkun, Yekta Gurlertop \\ Department of Cardiology, Trakya University, Edirne, Turkey \\ Correspondence to: Kenan Yalta, MD. Department of Cardiology, Trakya University, Edirne, Turkey. Email: kyalta@gmail.com. \\ Provenance: This is an invited Editorial commissioned by the Section Editor Yue Liu (The First Affiliated Hospital, Harbin Medical University, \\ Harbin, China). \\ Comment on: Feldman TE, Reardon MJ, Rajagopal V, et al. Effect of mechanically expanded vs self-expanding transcatheter aortic valve replacement \\ on mortality and major adverse clinical events in high-risk patients with aortic stenosis: the REPRISE III randomized clinical trial. JAMA \\ 2018;319:27-37.
}

Submitted Mar 05, 2018. Accepted for publication Apr 02, 2018.

doi: $10.21037 /$ cdt.2018.04.06

View this article at: http://dx.doi.org/10.21037/cdt.2018.04.06

In the recent years, transcatheter aortic valve implantation (TAVI) has gained a significant reputation as an alternative option for the management of aortic valve disease particularly in the setting of high-risk conditions for surgical aortic valve replacement (SAVR) (1-3). In the wellknown PARTNER-1 trial, TAVI was found to be associated with a significant mortality benefit in comparison to standard treatment among patients with aortic valve stenosis (AVS) (1). Interestingly, both TAVI and SAVR were demonstrated to have comparable safety and efficacy profiles along with similar all-cause mortality rates at 2 years in the recent NOTION study which was the first to enroll patients at a low-risk category as well (3). Similarly, SURTAVI (4) and PARTNER-2 (5) trials have approved the usefulness of TAVI as an alternative to SAVR among intermediaterisk patients leading to an expansion of TAVI indications in the recent guidelines for valvular heart disease (6). However, there still exists significant reservations about the feasibility of TAVI in low-risk patients who are traditionally deemed as perfect candidates for SAVR (7).

Given the proven efficacy of TAVI in clinical practice, transcatheter aortic bioprostheses have rapidly evolved in the recent years (8-10). Mechanistically, there currently exists three major types of bioprostheses in clinical use: balloon-expandable (BEV), self-expandable (SEV) and mechanically expanding valves (MEV) (11). However, there currently exists a relatively limited experience on MEVs in clinical practice $(8,11)$. Within this context, MEVs were implanted only in less than $10 \%$ of 27,760 cases undergoing TAVI between 2013 and 2016 according to a very recent multicentre registry comprising 79 centres (11). Similarly, clinical trials have mainly focused on the comparison between BEVs (Edwards SAPIEN/SAPIEN XT, etc.) and SEVs (Medtronic CoreValve, etc.) $(9,12)$. Mortality at 30 days and 1 year along with vascular and bleeding complications were found to be comparable between BEVs and SEVs in these studies $(9,13)$. However, SEVs were found to be associated with a lower device success as measured with higher rates of paravalvular leak (PVL) and the need for more than one valve in the same session along with a higher propensity for new-onset conduction disturbances requiring permanent pacemaker implantation (PPI) $(9,13)$. On the other hand, BEVs are more likely to elicit potentially fatal complications including annular rupture and coronary osteal occlusion particularly in the setting of certain risk factors $(8,11,14)$. Moreover, firstgeneration SEVs and BEVs are not amenable to valve repositioning or retrieval potentially leading to device malapposition along with device failure, PVL, coronary osteal occlusion and valve-in valve procedures in certain settings $(8,12)$. Taken together, both BEVs and SEVs harbor specific challenges in clinical practice particularly requiring higher device technologies (8).

On the other hand, MEVs harbor unique design and mechanical features potentially obviating the challenges associated with the first-generation balloon and self- 
expandable bioprostheses $(8,12,15,16)$. Within this context, the first experience with Sadra Lotus ${ }^{\mathrm{TM}}$ valve took place in $2007(8,12)$. This bioprosthesis primarily comprises a bovine pericardial tissue within a nitinol frame, and has a specific mechanism of deployment: the valve system loaded on the Lotus catheter is delivered through the native aortic valves $(8,12)$. At this point, once removing the outer layer of the catheter, the nitinol frame gets shortened in height, and enlarges radially with an increasing rigidity gradually transforming into a compressed state $(8,12,16)$. Of note, the device has a specific design allowing full retrieval and repositioning even just before the final release of the bioprosthesis potentially preventing device malapposition, device failure $(10,12)$. Since the valve starts to function in the earlier stages of deployment without any significant impediment to the transaortic blood flow, rapid ventricular pacing is not necessary during expansion $(8,12,16)$. This may pose a significant advantage in the setting of certain conditions including severe systolic or diastolic dysfunction and mitral stenosis, etc. where rapid pacing appears to be potentially detrimental. Importantly, the lower portion of the valve is girdled by an adaptive seal (consisting of polyurethane) that substantially minimizes PVL $(10,12)$.

Lotus valve has been tested in a variety of single-arm studies so far: REPRISE I (15), REPRISE II (16) and RESPOND (17) studies previously reported favorable outcomes including substantially reduced PVL and perfect valve hemodynamics at the cost of relatively high rates of permanent PPI in high-risk patients. On the other hand, Lotus valve was also compared with a variety of second generation valves including direct flow-medical (DFM) (18) and Edwards Sapien-3 (19) valves. Importantly, Lotus valve, despite having similar outcomes, was found to be associated with a higher incidence of PPI in these studies $(18,19)$.

In the recently published REPRISE III randomized clinical study, Feldman et al. compared Lotus valve with a SEV (Medtronic CoreValve) in terms of its efficacy and safety profiles in high-risk subjects undergoing TAVI (10). The study included 912 high-risk patients initially randomized to one of these valves in a ratio of 2:1 (607 with Lotus and 305 with CoreValve bioprostheses). Importantly, comparison of primary end points including composite safety outcomes at 1 month (all-cause mortality, major bleeding, vascular complications, moderate-severe kidney injury, etc.) as well as composite efficacy outcomes at 1 year (major stroke, all-cause mortality and moderatesevere PVL) demonstrated the non-inferiority of Lotus valve as compared with CoreValve (10). Moreover, Lotus valve exhibited a significantly superior profile particularly with regard to composite efficacy outcomes at 1 year. In particular, rate of moderate-severe PVL at 1-year which was the secondary end point of the study, appeared to be significantly reduced in the Lotus valve group as well (10). On the other, the study also yielded additional findings with important clinical implications that need to be further discussed within this context:

Firstly, incorporating an additional arm of BEV into the study would have yielded a more comparative and thorough view of the current device technology.

Secondly, the need for PPI was significantly higher in the Lotus valve group ( $35.5 \%$ vs. $19.6 \%$ at 1 month) in the study (10). This finding appears to be in line with previous single and double-arm studies (comparing Lotus valve with other valve types) (15-19). In other terms, one in every three patients undergoing TAVI with a Lotus valve needs a permanent PPI that still remains as the most significant challenge in this setting (20). In the REPRISE III trial, higher rate of PPI in the Lotus valve group was largely attributed, by the authors, to the close interaction of the valve structure with the left ventricular outflow tract (LVOT) and neighboring conducting system (10). Importantly, an implantation depth of $>5 \mathrm{~mm}$ and overstretching of LVOT (more than 10\%) due to annular oversizing was recently suggested as the fundamental predictors of new PPI among Lotus valve recipients (20). Accordingly, further data regarding these parameters might have been informative and interesting to the readers of this study (10). Importantly, the next generation Lotus valve namely Lotus Edge has been very recently put on the market (20). This latest generation is primarily equipped with Depth Guard ${ }^{\mathrm{TM}}$ system, an additional feature aiming to prevent the deep implantation of the device and hence; significantly reducing the need for PPI (20).

Thirdly, the rate of moderate-severe PVL at 1 year was found to be significantly lower in the Lotus valve group $(0.9 \%$ vs. $6.9 \%)$ (10). PVL is well known to be associated with severe calcification, malapposition as well as undersizing of the bioprosthetic valve, and appears to be a predictor of mortality after TAVI (10). However, half of the patients in the SEV group received a second generation SEV (Medtronic Evolut-R) starting midway during the study period, and the rate of moderate-severe PVL in these patients was reported to be $2.9 \%$ that was not statistically significant in comparison to Lotus valve recipients (10). Similarly, previous studies also reported no advantage of Lotus valve as compared with certain second-generation 


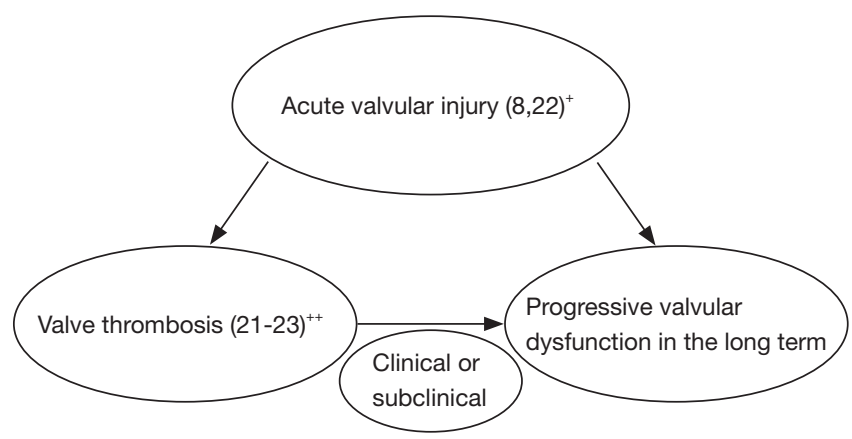

Figure 1 Potential association among acute valvular injury, valve thrombosis and chronic progressive valvular degeneration (dysfunction). ${ }^{+}$, potentially arises due to crimping, over or under expansion of the bioprosthesis and might be more likely to occur in the $\mathrm{MEVs} ;{ }^{++}$, mostly subclinical (asymptomatic and detectable only with advanced diagnostic modalities), occasionally clinical (grossly visible on conventional echocardiogram with or without signs and symptoms of heart failure, stroke, etc.) in the acute setting. MEVs, mechanically expanding valves.

BEVs and SEVs in terms of significant PVL $(18,19)$. Negation of Lotus valve superiority in this setting might be due to the small sample size of second-generation valves in these studies $(10,18,19)$. On the other hand, this may also imply that certain second-generation BEVs and SEVs, when positioned properly, have also favorable features in terms of PVL prevention, and may be considered to be on a par with the Lotus valve in this setting. Therefore, future studies are still warranted to confirm the absolute superiority of Lotus valve (also including the next generation Lotus Edge) in comparison to new generation SEVs and BEVs with regard to PVL prevention.

Fourthly, valve thrombosis in the Lotus valve group, though rare $(1.5 \%$ vs. $0 \%)$, has emerged as an interesting finding in this study (10). Evolution of valve thrombosis might have certain implications in the setting of TAVI: this phenomenon was previously suggested to be strongly associated with reduced valve motion as well as neurologic events including transient ischemic attacks (TIAs), stroke as well as acute heart failure (21-23). On the other hand, patients with valve thrombosis in the Lotus valve group did not incur any neurological event or mortality in the trial (10). However, overwhelming majority of bioprosthetic valve thromboses generally remain silent, and are not usually detectable on 2D-echocardiogram necessitating advanced diagnostic modalities including computed tomography (CT) $(21,23)$. Mechanistically, evolution of bioprosthetic valve thrombosis is largely attributed to the valvular traumatic injury associated with over or under expansion, or more likely "crimping" (process of compressing the valve into the delivery system) of the valve $(8,21)$. In a recent report of case series with early Lotus valve thrombosis, it was suggested that Lotus valve might be more prone to thrombosis largely due to the thicker metallic constituents of its stent frame potentially facilitating thrombogenesis at the aortic side of the leaflets (22). Regardless of suggested mechanisms, management of valve thrombosis is primarily based on anticoagulant therapy (warfarin) generally maintained for a couple of months, and rarely; surgery in the setting of cases unresponsive to anticoagulation $(22,23)$. More interestingly, persistence of valvular thrombus might potentially account for progressive valve degeneration and dysfunction in the chronic setting (21) possibly due to the fibrotic transformation of the organized thrombus.

In the long-term, progressive bioprosthetic valve degeneration also appears to be associated with valvular injury regardless of co-existing valve thrombosis. In particular, tighter crimping due to thicker leaflets (those comprising bovine pericardial tissue) and relatively small catheter sizes along with potential under expansion (as in the setting of Lotus valve (bovine pericardium) with a reportedly smaller area as compared with CoreValve (porcine pericardium) in the REPRISE III (10) might possibly be associated with a substantial damage within the valvular connective tissue ultimately leading to progressive bioprosthetic valve dysfunction in the long term (8). The potential association among acute valvular injury, valve thrombosis and chronic valvular degeneration is shown in Figure 1.

Taken together, the incidence of valve thrombosis (mostly subclinical) might have, in fact, been much higher in the Lotus valve group in the REPRISE III trial (10). Importantly, anticoagulant therapy (warfarin), in comparison to dual antiplatelet therapy (DAPT), was suggested as a more effective strategy for the prevention this phenomenon as well (21). Therefore, despite the lack of recommendation in the current guidelines on valvular heart disease (24), routine use of warfarin for a certain duration, where possible, on top of antiplatelet regimens may be recommended in certain TAVI patients including Lotus valve recipients $(21,22)$. Since, patients with Lotus valve might also have a speculative proclivity for chronic bioprosthetic dysfunction, a longer follow-up may be necessary for patients in the REPRISE III trial.

Fifthly, stroke was reported to be less frequent $(7.0 \%$ 


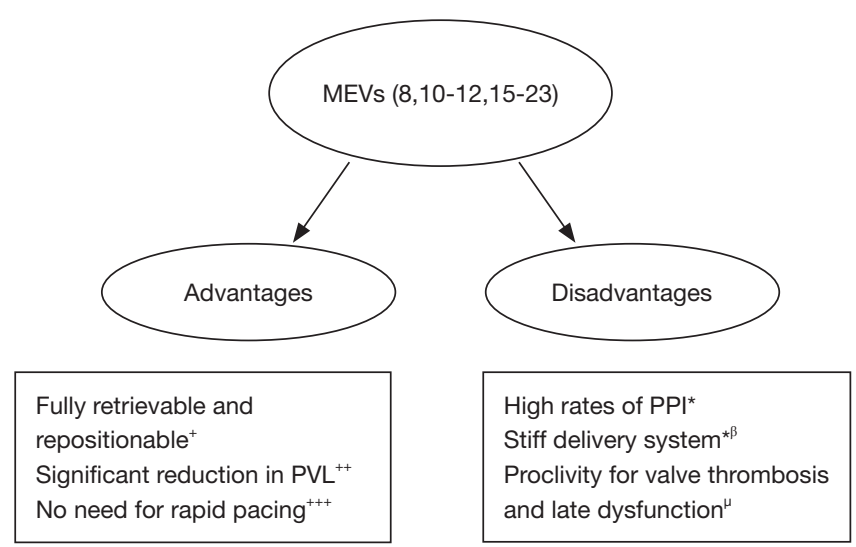

Figure 2 Summary of pros and cons of MEVs. ${ }^{+}$, potentially preventing device embolization and failure (requiring valve-in valve procedures) as well as device malapposition associated with coronary osteal occlusion, PVL and PPI; ${ }^{++}$, in comparison to first-generation balloon and self-expandable valves; ${ }^{+++}$, poses a significant advantage in the setting of severe systolic or diastolic heart failure at baseline; *, significantly improved in the nextgeneration valves; ${ }^{\beta}$, might potentially be associated with procedural aortic dissection as well as embolism; ${ }^{\mu}$, still remains as a speculative phenomenon that might be associated with tighter crimping as well as under expansion. MEVs, mechanically expandable valves; PVL, paravalvular leak; PPI, permanent pacemaker implantation.

vs. 9.4\%) in the Lotus valve group (10). In the setting of TAVI, cerebrovascular embolism may potentially originate from a variety of sources including calcified native valves, new clot formation as well as atherosclerotic plaques in the proximal aorta. According to a recent European registry, first generation Lotus valve, as compared with other valve types, was found to be associated with a higher incidence of procedural aortic dissection largely due to its stiff delivery system (11). Therefore, stiff delivery system might, to a large extent, have accounted for the cerebrovascular embolism particularly originating from the aorta in the Lotus valve group (10). Therefore, it seems reasonable to avoid TAVI with a first-generation Lotus valve in the setting of poor aortic features including aneurysm (11), mural thrombus, heavy calcification or atherosclerosis, etc. Importantly, Lotus Edge, the second-generation Lotus valve, has a lower profile and more resilient delivery system (20) that might lead to further declines in stroke incidence among patients undergoing TAVI.

And lastly, it should be remembered that Lotus valve was temporarily recalled from the market due to a pin problem in its locking mechanism in the early 2017 (20). It might have been interesting to discuss, in a detailed manner, such device-related problems that had occurred in the Lotus valve group (if any) as well as the attitude of the authors (10) towards these mechanical challenges during the procedure. An overview of pros and cons of MEVs are summarized in Figure 2.

In conclusion, the authors (10) should be congratulated for their well-designed trial that, besides substantiating previous studies on MEVs (15-19), shed light on further clinical implications of Lotus valve implantation in the setting of TAVI. However, future studies are still warranted to investigate MEVs (particularly the next generation ones) with regard to their safety and efficacy profiles as compared with other valve types in the clinical setting.

\section{Acknowledgements}

None.

\section{Footnote}

Conflicts of Interest: The authors have no conflicts of interest to declare.

\section{References}

1. Kapadia SR, Leon MB, Makkar RR, et al. PARTNER trial investigators. 5-year outcomes of transcatheter aortic valve replacement compared with standard treatment for patients with inoperable aortic stenosis (PARTNER-1): a randomised controlled trial. Lancet 2015;385:2485-91.

2. Yalta K, Kaya Ç, Aksoy Y, et al. Acute myocardial infarction shortly after valve-in-valve transcatheter aortic valve implantation successfully managed with challenging percutaneous coronary intervention. Turk Kardiyol Dern Ars 2018;46:61-5.

3. Søndergaard L, Steinbrüchel DA, Ihlemann N, et al. Two-year outcomes in patients with severe aortic valve stenosis randomized to transcatheter versus surgical aortic valve replacement: the all-comers Nordic aortic valve intervention randomized clinical trial. Circ Cardiovasc Interv 2016;9. pii:e003665.

4. Reardon MJ, Van Mieghem NM, Popma JJ, et al. SURTAVI investigators. Surgical or Transcatheter AorticValve Replacement in Intermediate-Risk Patients. N Engl J Med 2017;376:1321-31.

5. Leon MB, Smith CR, Mack MJ, et al. Transcatheter or 
Surgical Aortic-Valve Replacement in Intermediate-Risk Patients. N Engl J Med 2016;374:1609-20.

6. Baumgartner H, Falk V, Bax JJ, et al. 2017 ESC/EACTS Guidelines for the management of valvular heart disease. Eur Heart J 2017;38:2739-91.

7. Arora S, Vavalle JP. Transcatheter aortic valve replacement in intermediate and low risk patients-clinical evidence. Ann Cardiothorac Surg 2017;6:493-7.

8. Fanning JP, Platts DG, Walters DL, et al. Transcatheter aortic valve implantation (TAVI): valve design and evolution. Int J Cardiol 2013;168:1822-31.

9. Abdel-Wahab M, Mehilli J, Frerker C, et al. Comparison of balloon-expandable vs self-expandable valves in patients undergoing transcatheter aortic valve replacement: the CHOICE randomized clinical trial. JAMA 2014;311:1503-14.

10. Feldman TE, Reardon MJ, Rajagopal V, et al. Effect of mechanically expanded vs self-expanding transcatheter aortic valve replacement on mortality and major adverse clinical events in high-risk patients with aortic stenosis: the REPRISE III randomized clinical trial. JAMA 2018;319:27-37.

11. Eggebrecht H, Vaquerizo B, Moris C, et al. Incidence and outcomes of emergent cardiac surgery during transfemoral transcatheter aortic valve implantation (TAVI): insights from the European Registry on Emergent Cardiac Surgery during TAVI (EuRECSTAVI). Eur Heart J 2018;39:676-84.

12. Buellesfeld L, Gerckens U, Grube E. Percutaneous implantation of the first repositionable aortic valve prosthesis in a patient with severe aortic stenosis. Catheter Cardiovasc Interv 2008;71:579-84.

13. Chieffo A, Buchanan GL, Van Mieghem NM, et al. Transcatheter aortic valve implantation with the Edwards SAPIEN versus the Medtronic CoreValve Revalving system devices: a multicenter collaborative study: the PRAGMATIC Plus Initiative (Pooled-RotterdAmMilano-Toulouse In Collaboration). J Am Coll Cardiol 2013;61:830-6.

14. Soufras GD, Hahalis G, Kounis NG. Thrombotic complications associated with transcatheter aortic valve implantation: the role of Kounis hypersensitivity-associated thrombotic syndrome. Cardiovasc Pathol 2014;23:383-4.

15. Meredith IT, Worthley SG, Whitbourn RJ, et al. Transfemoral aortic valve replacement with the repositionable Lotus Valve System in high surgical risk patients: the REPRISE I study. EuroIntervention 2014;9:1264-70.
16. Meredith IT, Walters DL, Dumonteil N, et al. 1-Year Outcomes with the fully repositionable and retrievable lotus transcatheter aortic replacement valve in 120 highrisk surgical patients with severe aortic stenosis: results of the REPRISE II study. JACC Cardiovasc Interv 2016;9:376-84.

17. Falk V, Wöhrle J, Hildick-Smith D, et al. Safety and efficacy of a repositionable and fully retrievable aortic valve used in routine clinical practice: the RESPOND Study. Eur Heart J 2017;38:3359-66.

18. Giannini F, Latib A, Montorfano M, et al. A comparison of the fully repositionable and retrievable Boston Lotus and direct flow medical valves for the treatment of severe aortic stenosis: A single center experience. Catheter Cardiovasc Interv 2018;91:966-74.

19. Seeger J, Gonska B, Rottbauer W, et al. Outcome with the repositionable and retrievable boston scientific lotus valve compared with the balloon-expandable Edwards sapien 3 valve in patients undergoing transfemoral aortic valve replacement. Circ Cardiovasc Interv 2017;10. pii:e004670.

20. Pellegrini C, Hengstenberg C, Husser O. The Lotus dilemma-respond to paravalvular leakage, but not answering pacemaker implantations? J Thorac Dis 2017;9:2804-7.

21. Chakravarty T, Søndergaard L, Friedman J, et al. Subclinical leaflet thrombosis in surgical and transcatheter bioprosthetic aortic valves: an observational study. Lancet 2017;389:2383-92.

22. Salido-Tahoces L, Hernandez-Antolin RA, FernándezGolfin C, et al. Three Cases of Early Lotus Valve Thrombosis. JACC Cardiovasc Interv 2016;9:983-6.

23. Schoechlin S, Ruile P, Neumann FJ, et al. Early hypoattenuated leaflet thickening and restricted leaflet motion of a Lotus transcatheter heart valve detected by 4D computed tomography angiography. EuroIntervention 2015;11:e1.

24. Nishimura RA, Otto CM, Bonow RO, et al. 2017 AHA/ ACC Focused Update of the 2014 AHA/ACC Guideline for the Management of Patients With Valvular Heart Disease: A Report of the American College of Cardiology/ American Heart Association Task Force on Clinical Practice Guidelines. Circulation 2017;135:e1159-95.

Cite this article as: Yalta K, Gurdogan M, Zorkun C, Gurlertop Y. Mechanically expanding transcatheter aortic valves: pros and cons of a unique device technology. Cardiovasc Diagn Ther 2018;8(4):538-542. doi: 10.21037/cdt.2018.04.06 